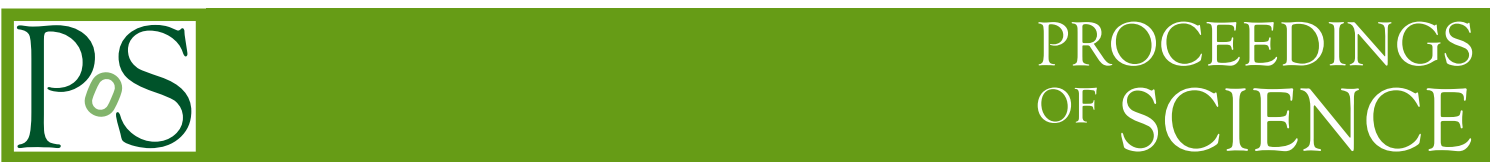

\title{
Theoretical Status of Rare B Decays with Muons
}

\section{Christoph Bobeth}

IPHC, Université de Strasbourg, CNRS/IN2P3, F-67037, Strasbourg, France

\section{Gudrun Hiller*i and Giorgi Piranishvili}

Institut für Physik, Technische Universität Dortmund, D-44221 Dortmund, Germany

\begin{abstract}
Rare $B$ decays allow to investigate fundamental interactions regarding their flavor, chiral, Dirac and $\mathrm{CP}$ properties. In anticipation of the large data samples of exclusive $B$ decays into muons from the forthcoming $\mathrm{LHC}$ experiments, in particular $\mathrm{LHCb}$, as well as possible super flavor factories, we review the theoretical status and outline future opportunities to explore the borders of the Standard Model and beyond.
\end{abstract}

12th International Conference on B-Physics at Hadron Machines September 7-11, 2009

Heidelberg, Germany.

\footnotetext{
*Speaker.

${ }^{\dagger}$ Preprint DO-TH 09/18
} 


\section{Introduction}

The quest for physics Beyond the Standard Model (BSM) is of highest priority at current and future flavor facilities. We focus here on exclusive semileptonic decays induced by $b \rightarrow s l^{+} l^{-}$ transitions to test the Standard Model (SM) and probe BSM physics. The decays into muons $l=\mu$ are especially well suited for investigations at hadron colliders, such as the Tevatron and most important in terms of high luminosity, the Large Hadron Collider (LHC). The observables presented are also of relevance to super flavor $e^{+} e^{-}$facilities.

\section{A brief $b \rightarrow s \mu^{+} \mu^{-}$primer}

We review briefly the current experimental situation for rare $b \rightarrow s l^{+} l^{-}$processes, recap the theory framework how to extract BSM couplings from $\Delta B=1$ observables, and comment on cuts.

\subsection{The experimental situation}

Important modes for hadron colliders are the exclusive decays $B \rightarrow K^{(*)} \mu^{+} \mu^{-}, B_{s} \rightarrow \Phi \mu^{+} \mu^{-}$ and $\Lambda_{b} \rightarrow \Lambda \mu^{+} \mu^{-}$with SM branching ratios of the order $\mathscr{B}_{S M} \sim 10^{-7}-10^{-6}$. The decays $B \rightarrow$ $K \mu^{+} \mu^{-}$and $B \rightarrow K^{*} \mu^{+} \mu^{-}$have been observed at the $B$ factory experiments Belle and BaBar [1]

$$
\mathscr{B}\left(B \rightarrow K \mu^{+} \mu^{-}\right)=(0.48 \pm 0.06) \cdot 10^{-6}, \quad \mathscr{B}\left(B \rightarrow K^{*} \mu^{+} \mu^{-}\right)=\left(1.15_{-0.15}^{+0.16}\right) \cdot 10^{-6},
$$

in agreement with the SM. The Tevatron is close to seeing $\mathscr{B}\left(B_{s} \rightarrow \Phi \mu^{+} \mu^{-}\right)$[2]. Experimental investigations of more involved (and also more BSM diagnostical) observables such as dilepton mass spectra, lepton angle distributions and dimuon to dielectron ratios are currently underway $[4,5,6]$, see Sec 2.4 and 3 . The purely leptonic decay $B_{s} \rightarrow \mu^{+} \mu^{-}$is very rare in the SM, $\mathscr{B}_{S M} \simeq$ $3 \cdot 10^{-9}$, but can be significantly enhanced in models that circumvent the lepton mass suppression present in the SM. Currently a bound only exists for its branching ratio $\mathscr{B}\left(B_{S} \rightarrow \mu^{+} \mu^{-}\right)<3.6$. $10^{-8} @ 90 \%$ C.L. [3]. Frequently averages over $e^{+} e^{-}$and $\mu^{+} \mu^{-}$final states are quoted for the semileptonic $b \rightarrow s l^{+} l^{-}$observables. Indeed the SM predictions agree up to very small corrections after appropriate cuts in the dilepton mass $q^{2}$ have been taken into account [7]. However, the averaging washes out possible lepton flavor non-universal effects, such as from Higgs exchanges, leptoquarks or R-parity violation, and therefore applies only to a restricted set of BSM models. So far inclusive decays into dimuons have not been observed (at $\geq 5 \sigma$ ) yet, however, the lepton average $(l=e, \mu)$ is observed $\mathscr{B}\left(B \rightarrow X_{s} l^{+} l^{-}\right)=\left(3.66_{-0.77}^{+0.76}\right) \cdot 10^{-6}$ for $q^{2}>0.04 \mathrm{GeV}^{2}[1]$.

\subsection{The effective theory}

Our aim is to test the SM and probe BSM with quantum loop effects. The framework used is a generalized Fermi theory of electroweak interactions valid for external momenta much below the scale where electroweak interactions, e.g., for $B$ physics $m_{b}^{2} \ll m_{W}^{2}$, are induced

$$
\mathscr{H}_{\mathrm{eff}}=-4 \frac{G_{F}}{\sqrt{2}} V_{t b} V_{t s}^{*} \sum_{i} C_{i}(\mu) O_{i}(\mu)
$$

A picture of matching the full theory, $\mathscr{L}$, onto the effective one, Eq. (2.2), is given in Fig. 1 for the SM. New Physics (NP) can appear in the Wilson coefficients $C_{i}=C_{i}^{\mathrm{SM}}+C_{i}^{\mathrm{NP}}$ or in new operators 

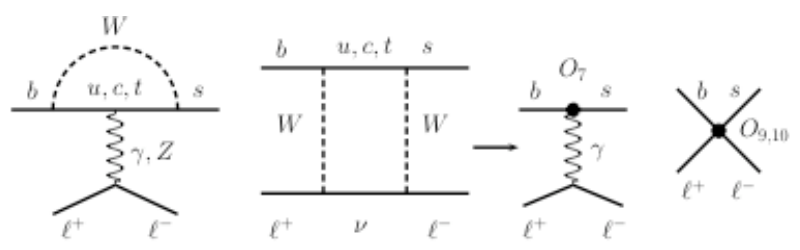

Figure 1: Lowest order Feynman diagrams in the SM contributing to $b \rightarrow s l^{+} l^{-}$in the full (left) and effective theory (right).

\begin{tabular}{c|c|c|c} 
Wilson coefficient & description & SM & enhancement in models \\
\hline$C_{1,2}$ & charged current & YES & \\
\hline$C_{3, ., 6}$ & QCD penguins & YES & SUSY \\
$C_{7,8}$ & $\gamma, g$-dipole & YES & SUSY, large tan $\beta$ \\
$C_{9,10}$ & (axial-)vector & YES & SUSY \\
\hline$C_{S, P}$ & (pseudo-)scalar & $\sim m_{l} m_{b} / m_{W}^{2}$ & SUSY, large tan $\beta$, R-parity viol. \\
$C_{S, P}^{\prime}$ & (pseudo-)scalar flipped & $\sim m_{l} m_{s} / m_{W}^{2}$ & SUSY, R-parity viol. \\
$C_{3, \ldots, 6}^{\prime}$ & QCD peng. flipped & $\sim m_{s} / m_{b}$ & SUSY \\
$C_{7,8}^{\prime}$ & $\gamma, g$-dipole flipped & $\sim m_{s} / m_{b}$ & SUSY, esp. large tan $\beta$ \\
$C_{9,10}^{\prime}$ & (axial-)vector flipped & $\sim m_{s} / m_{b}$ & SUSY \\
$C_{T, T 5}$ & tensor & negligible & leptoquarks
\end{tabular}

Table 1: Effective couplings for $b \rightarrow s l^{+} l^{-}$and appearance in various models, see, e.g., [9]. The flipped operators $O_{i}^{\prime}$ are obtained from the $O_{i}$ by interchanging the chiralities $L \leftrightarrow R$.

$O_{i}$. The effective theory framework allows for a model-independent analysis to determine the $C_{i}$ from multi-observables/multi-processes [8]. Couplings for $b \rightarrow s l^{+} l^{-}$are given in Table 1. Within the SM the decays $b \rightarrow s l^{+} l^{-}$are well described by ten operators with real coefficients. In general, the number of operators consistent with gauge and Poincare invariance is more than twice as large. The Wilson coefficients can also carry CP phases. In addition $b \rightarrow s l^{+} l^{-}$transitions depend in general on the lepton flavor, i.e., $C_{i} \rightarrow C_{i}^{l}$, and even lepton flavor violation maybe considered with the additional operators $O_{i}^{l, l^{\prime}} \sim \bar{s} \Gamma b \bar{l} \Gamma^{\prime} l^{\prime}$. Since the resulting number of orthogonal observables required for an extraction of the complete effective operator structure in $\mathscr{H}_{\text {eff }}$ is very large, constrained model frameworks are useful, such as minimal flavor violation, where $C_{i}^{\prime} / C_{i} \sim m_{s} / m_{b}$.

\subsection{Dilepton invariant mass cuts}

Kinematical cuts are vital to reduce the background from intermediate charmonia via $b \rightarrow$ $s \Psi^{(n)}\left(\rightarrow l^{+} l^{-}\right) \rightarrow s l^{+} l^{-}$. One distinguishes the low $q^{2}$ region with $q^{2}<m_{J / \Psi}^{2}$ and the high $q^{2}$ region with $q^{2}>m_{\Psi^{\prime}}^{2}$. Another important reason to use cuts is that there is no single rigorous theory framework available for exclusive $b \rightarrow s l^{+} l^{-}$decays in the whole kinematical region. Only $q^{2}$-binned data allow for a systematic comparison with theory. Theoretically preferred is the low dilepton mass below the $J / \Psi$, where many works exist, e.g., [10]. The high $q^{2}$ region is calculable with an $1 / \sqrt{q^{2}}, 1 / m_{b}$ expansion [11]. The whole $q^{2}$ region tests the SM, and different regions are sensitive to different NP couplings and models. 


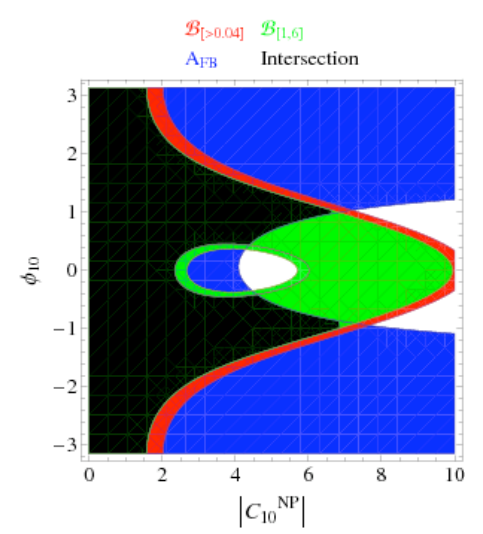

Figure 2: Constraints on the magnitude and $\mathrm{CP}$ phase of the NP contribution to $C_{10}$ using different, complementary measurements. The black area is allowed by $\mathscr{B}$ (green, red) and $A_{\mathrm{FB}}$ (blue) constraints [12].

\subsection{Forward-backward asymmetry and early data}

Consider the forward-backward asymmetry $A_{\mathrm{FB}}$ in $B \rightarrow K^{*} l^{+} l^{-}$decays. The low $q^{2}$ region is sensitive to $\operatorname{sign} C_{7}$, whereas the high $q^{2}$ region probes the 4-Fermi operators, $\operatorname{sign} C_{9}^{*} C_{10}$. Recent data strongly favor the sign of $A_{\mathrm{FB}}$ in the high $q^{2}$ region to be SM-like: $A_{\mathrm{FB}}^{\text {high }} q^{2}=0.76_{-0.32}^{+0.52} \pm 0.07$ (BaBar) [4], $A_{\mathrm{FB}}^{\text {high } q^{2}>16 \mathrm{GeV}^{2}}=0.66_{-0.16}^{+0.11} \pm 0.04$ (Belle) [6]. Already fixing $\operatorname{sign} A_{\mathrm{FB}}^{\text {high }} q^{2}>0$ yields useful constraints which are orthogonal to the ones from the branching ratio measurements [12], see Fig. 2. Here the allowed region of the phase and magnitude of the NP contribution to $C_{10}$ is shown. Note that $O_{10} \sim \bar{s}_{L} \gamma_{\mu} b_{L} \bar{l} \gamma^{\mu} \gamma_{5} l$ captures the effect of non-standard $b Z s$-penguins, while their contribution to $O_{9} \sim \bar{s}_{L} \gamma_{\mu} b_{L} \bar{l} \gamma^{\mu} l$ is suppressed by $\left(1-4 \sin ^{2} \theta_{W}\right) \ll 1[13]$.

\section{The future: Angular analysis}

After observing the $B \rightarrow K^{(*)} l^{+} l^{-}$decays and early measurements of their asymmetries [4, 5, 6] one can use these modes for detailed investigations of the $b \rightarrow s$ transitions and the structure of $\mathscr{H}_{\text {eff }}$, Eq. (2.2). A multitude of complementary observables can be obtained from the angular distributions in $B \rightarrow K^{*}(\rightarrow K \pi) l^{+} l^{-}$[12],[14]-[17], in $B_{s} \rightarrow \Phi(\rightarrow K K) l^{+} l^{-}$[12], and the simpler $B \rightarrow K l^{+} l^{-}$decays [9]. $\Lambda_{b} \rightarrow \Lambda$ decays offer further possibilities through polarization studies [18].

\section{1 $B \rightarrow K^{*}(\rightarrow K \pi) l^{+} l^{-}$}

The full differential decay distribution (in $K^{*}$-zero-width approximation) can be written as

$$
d^{4} \Gamma=\frac{3}{8 \pi} J\left(q^{2}, \theta_{l}, \theta_{K^{*}}, \phi\right) d q^{2} d \cos \theta_{l} d \cos \theta_{K^{*}} d \phi,
$$

where

$$
\begin{aligned}
J\left(q^{2}, \theta_{l}, \theta_{K^{*}}, \phi\right) & =J_{1}^{s} \sin ^{2} \theta_{K^{*}}+J_{1}^{c} \cos ^{2} \theta_{K^{*}}+\left(J_{2}^{s} \sin ^{2} \theta_{K^{*}}+J_{2}^{c} \cos ^{2} \theta_{K^{*}}\right) \cos 2 \theta_{l} \\
& +J_{3} \sin ^{2} \theta_{K^{*}} \sin ^{2} \theta_{l} \cos 2 \phi+J_{4} \sin 2 \theta_{K^{*}} \sin 2 \theta_{l} \cos \phi+J_{5} \sin 2 \theta_{K^{*}} \sin \theta_{l} \cos \phi \\
& +J_{6} \sin ^{2} \theta_{K^{*}} \cos \theta_{l}+J_{7} \sin 2 \theta_{K^{*}} \sin \theta_{l} \sin \phi \\
& +J_{8} \sin 2 \theta_{K^{*}} \sin 2 \theta_{l} \sin \phi+J_{9} \sin ^{2} \theta_{K^{*}} \sin ^{2} \theta_{l} \sin 2 \phi, \quad J_{i}=J_{i}\left(q^{2}\right) .
\end{aligned}
$$


Here, $\theta_{l}$ denotes the angle between the $l^{-}$and the $\bar{B}$ in the dilepton center-of-mass system (CMS $)^{1}$, $\theta_{K^{*}}$ is the angle between the $K$ and the $\bar{B}$ in the $K^{*}$-CMS and $\phi$ is the angle between the normals of the $K \pi$ and the $l^{+} l^{-}$plane. The angular distribution $d^{4} \bar{\Gamma}$ of the CP-conjugate decays is obtained after flipping the sign of the CP phases and by replacing $J_{1,2,3,4,7} \rightarrow \bar{J}_{1,2,3,4,7}$ and $J_{5,6,8,9} \rightarrow-\bar{J}_{5,6,8,9}$. The familiar $B \rightarrow K^{*} l^{+} l^{-}$observables can be recovered as $\Gamma \sim J_{1}-J_{2} / 3, A_{\mathrm{FB}} \sim J_{6}, A_{T}^{(2)} \sim J_{3}[15]$, $A_{\mathrm{CP}} \sim \Gamma-\bar{\Gamma}$ and the forward-backward $\mathrm{CP}$ asymmetry $A_{\mathrm{FB}}+\bar{A}_{\mathrm{FB}} \sim A_{\mathrm{FB}}^{\mathrm{CP}}[13]$. The angular analysis makes many more observables available. Besides additional CP asymmetries [12], discussed in Sec 3.2, further transverse asymmetries $A_{T}^{(3)}, A_{T}^{(4)}$ have been proposed as "simple, clean, sensitive, precise" probes of the dipole couplings $C_{7}^{(\prime)}$ [16]. Some angular observables exhibit features known from $A_{\mathrm{FB}}$ in the sense that a zero is present in the SM which can shift or go away in the presence of NP. Studies in many BSM models are performed in Ref. [17].

\subsection{CP asymmetries}

$\mathrm{CP}$ asymmetries in $b \rightarrow s$ transitions are doubly Cabibbo-suppressed $A_{i} \propto \operatorname{Im}\left[V_{u b} V_{u s}^{*} / V_{t b} V_{t s}^{*}\right] \sim$ $10^{-2}$ in the SM and any model where CP and flavor violation stems solely from the Yukawa matrices. Experimental investigations of the $A_{i}$ are important tests of this paradigm. From $d^{4} \Gamma$ and $d^{4} \bar{\Gamma}$ one can construct eight CP asymmetries $A_{i} \propto J_{i}-\bar{J}_{i}$ [14], sensitive to different Wilson coefficients [12]. The $A_{3,9}$ vanish in the SM by helicity conservation. They are sensitive to right-handed currents $C_{i}^{\prime}$. The $A_{3,9,(6)}$ can be extracted from a single-differential distribution in $\phi\left(\theta_{l}\right)$. The $A_{7,8,9}$ are T-odd and receive no suppression by small strong phases, such as those predicted by QCD factorization at low $q^{2}[10]$. The $A_{5,6,8,9}$ are CP-odd and can be extracted without tagging from $\Gamma+\bar{\Gamma}$. Both $A_{7}$ and $A_{6}$ are sensitive to $Z$-penguins $\left(\sim C_{10}\right)$. While T-even CP asymmetries $\propto \sin \Delta_{S} \sin \Delta_{W}$ vanish for small strong phases $\Delta_{S}$, the T-odd asymmetries $\propto \cos \Delta_{S} \sin \Delta_{W}$ exhibit maximal sensitivity to the CP phases $\Delta_{W}$ in this limit. Contrary to the other $A_{i}$, the T-odd asymmetries $A_{7,8}$ and $A_{9}$ can be order one with NP (integrated over low $q^{2}$ ) [12], see Fig. 3. In each plot all other NP Wilson coefficients have been set to zero, and $B$ physics constraints have been taken into account.

$3.3 B_{s} \rightarrow \Phi(\rightarrow K K) l^{+} l^{-}$

The angular distributions in $B_{s} \rightarrow \Phi(\rightarrow K K) l^{+} l^{-}$allow to study CP violation in interference between decay and $B_{s}$ mixing. With the CP-odd asymmetries $A_{5,6,8,9}$ this is possible without flavor-tagging [12]. (Unlike $\bar{B}_{d}, B_{d} \rightarrow K^{*}\left(\rightarrow K^{\mp} \pi^{ \pm}\right) l^{+} l^{-}$or charged $B$ decays, $B_{s} \rightarrow \Phi$ is not self-tagging). The angular distribution of $B_{s} \rightarrow \Phi(\rightarrow K K) l^{+} l^{-}$is analogously defined as the one of $B \rightarrow K^{*}(\rightarrow K \pi) l^{+} l^{-}$decays. Dominant differences between the decay amplitudes originate from $S U(3)_{F}$ breaking. The biggest effects such as those from form factors, decay constants are expected to cancel in the asymmetries. Significant differences between $B \rightarrow K^{*}$ and $B_{s} \rightarrow \Phi$ observables arise from the mixing properties. $B_{s}-\bar{B}_{s}$ mixing has a substantial width difference $\Delta \Gamma_{s} /(2 \Gamma) \sim \mathscr{O}(0.1)$ and allows to measure time-integrated $\mathrm{CP}$ asymmetries, sensitive also to the $B_{s}$ mixing phase [12].

\section{4 $B \rightarrow K l^{+} l^{-}$}

The full angular distribution in $B \rightarrow K l^{+} l^{-}$decays can be written in terms of the decay rate

\footnotetext{
${ }^{1}$ Note that the lepton angle is also frequently defined w.r.t. the $l^{+}$, and $\theta_{l}\left(\bar{B} l^{-}\right)=\pi-\theta_{l}\left(\bar{B} l^{+}\right)$. Here, $\bar{B} \equiv b \bar{q}$.
} 

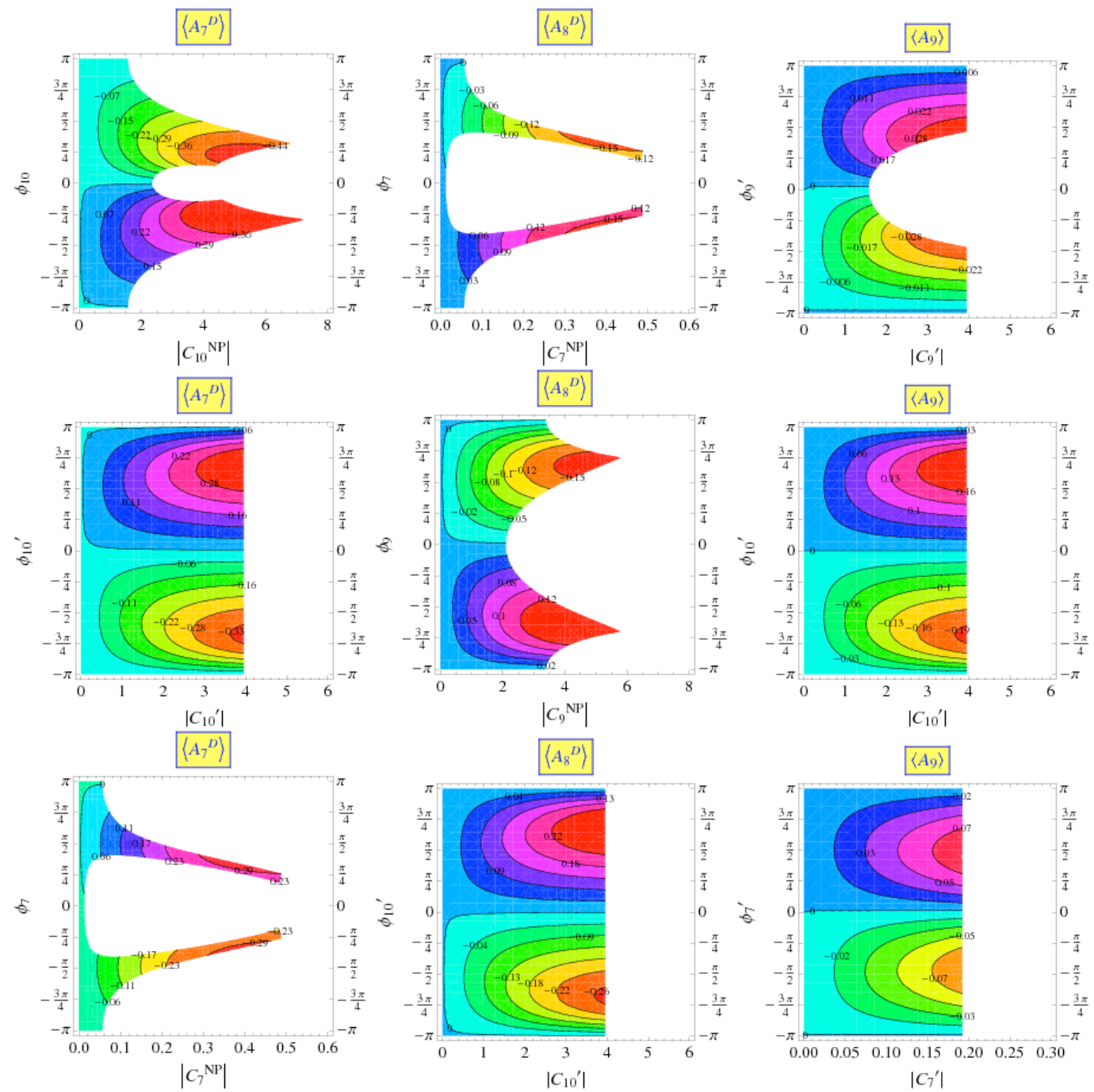

Figure 3: The (low $q^{2}$ integrated) T-odd CP asymmetries $A_{7,8}$ and $A_{9}$ depending on NP Wilson coefficients after applying experimental constraints. Figures adopted from [12, 19].

$\Gamma^{l}$, the forward-backward asymmetry $A_{\mathrm{FB}}^{l}$ and a flat term, $F_{H}^{l}$, as [9]

$$
\frac{1}{\Gamma^{l}} \frac{d \Gamma^{l}}{d \cos \theta_{l}}=\frac{3}{4}\left(1-F_{H}^{l}\right)\left(1-\cos ^{2} \theta_{l}\right)+F_{H}^{l} / 2+A_{\mathrm{FB}}^{l} \cos \theta_{l}
$$

Here, the dependence on the lepton species $l$ is kept to allow for non-universal phenomena. Other observables to probe such effects are $R_{H}=\mathscr{B}\left(B \rightarrow H \mu^{+} \mu^{-}\right) / \mathscr{B}\left(B \rightarrow H e^{+} e^{-}\right), H=K^{(*)}, X_{S}$ [7]. In the $\mathrm{SM}$ the observables $F_{H}^{l}, A_{\mathrm{FB}}^{l}$ and $R_{K}-1$ are strongly suppressed by the lepton mass and very small, and $\Gamma_{\mathrm{SM}}^{l} \propto \sin ^{2} \theta_{l}$ [7,9]. Sizeable BSM effects are possible (here for low $q^{2}$ ) [9]

$$
\left|A_{\mathrm{FB}}^{e}\right|<13 \%, \quad\left|A_{\mathrm{FB}}^{\mu}\right|<15 \%, \quad R_{K}-1=\mathscr{O}(1), \quad F_{H}^{e, \mu}<\mathscr{O}(0.5) .
$$

It follows that the forward-backward asymmetry of $B \rightarrow K l^{+} l^{-}$cannot be neglected in a modelindependent way. Correlations between the angular observables and $R_{K}$ and further $b \rightarrow s l^{+} l^{-}$ observables in several BSM scenarios are worked out in Ref. [9]. 


\section{Summary}

The coming years bring us large samples of flavor physics data from the LHC. This way comes into reach a multitude of observables from exclusive $b \rightarrow s \mu^{+} \mu^{-}$processes, which allow to precisely map out the structure of the underlying physics. Decays specific to super flavor factories into dielectrons $b \rightarrow s e^{+} e^{-}$and also ditau and dineutrino modes provide further, complementary information. Quark flavor hierarchies in the SM predict $b \rightarrow d$ transitions to be suppressed with respect to $b \rightarrow s$ ones, in agreement with the observed values of $\Delta m_{d, s}$ and the $b \rightarrow(s, d) \gamma$ rates. It is open to test this CKM-feature for semileptonic decays as well, where first data on $B \rightarrow \pi l^{+} l^{-}$ [20] have just become available. My favorite semi-near term questions for $B$ decays with $l^{+} l^{-}$are: $A_{\mathrm{FB}}$ (at low $\left.q^{2}\right), \mathscr{B}\left(B_{s} \rightarrow \mu^{+} \mu^{-}\right), R_{K, X_{s}}$ (improved), $F_{H}^{l}, \mathscr{B}\left(B_{d} \rightarrow \mu^{+} \mu^{-}\right) / \mathscr{B}\left(B_{s} \rightarrow \mu^{+} \mu^{-}\right), A_{i(T)}$.

\section{Acknowledgments}

This work is supported in part by the Bundesministerium für Bildung und Forschung (BMBF) and the German-Israeli-Foundation (G.I.F.).

\section{References}

[1] E. Barberio et al. [Heavy Flavor Averaging Group], arXiv:0808.1297 [hep-ex]. Online http://www.slac.stanford.edu/xorg/hfag from September 2009.

[2] Talk by M.J.Morello, this conference.

[3] Public CDF note 9892.

[4] B. Aubert et al. [BABAR Collab.], Phys. Rev. D 79 (2009) 031102 [arXiv:0804.4412 [hep-ex]].

[5] B. Aubert et al. [BABAR Collab.], Phys. Rev. Lett. 102, 091803 (2009) [arXiv:0807.4119 [hep-ex]].

[6] J. T. Wei et al. [BELLE Collab.], Phys. Rev. Lett. 103, 171801 (2009) [arXiv:0904.0770 [hep-ex]].

[7] G. Hiller and F. Kruger, Phys. Rev. D 69, 074020 (2004) [arXiv:hep-ph/0310219].

[8] A. Ali, G. F. Giudice and T. Mannel, Z. Phys. C 67, 417 (1995) [arXiv:hep-ph/9408213].

[9] C. Bobeth, G. Hiller and G. Piranishvili, JHEP 0712 (2007) 040 [arXiv:0709.4174 [hep-ph]].

[10] M. Beneke, T. Feldmann and D. Seidel, Eur. Phys. J. C 41 (2005) 173 [arXiv:hep-ph/0412400].

[11] B. Grinstein and D. Pirjol, Phys. Rev. D 70 (2004) 114005 [arXiv:hep-ph/0404250].

[12] C. Bobeth, G. Hiller and G. Piranishvili, JHEP 0807 (2008) 106 [arXiv:0805.2525 [hep-ph]].

[13] G. Buchalla, G. Hiller and G. Isidori, Phys. Rev. D 63 (2000) 014015 [arXiv:hep-ph/0006136].

[14] F. Kruger et al., Phys. Rev. D 61 (2000) 114028 [Erratum-ibid. D 63 (2001) 019901] [arXiv:hep-ph/9907386].

[15] F. Kruger and J. Matias, Phys. Rev. D 71 (2005) 094009 [arXiv:hep-ph/0502060].

[16] U. Egede et al., JHEP 0811 (2008) 032 [arXiv:0807.2589 [hep-ph]].

[17] W. Altmannshofer et al., JHEP 0901 (2009) 019 [arXiv:0811.1214 [hep-ph]].

[18] T. M. Aliev, A. Ozpineci and M. Savci, Phys. Rev. D 67, 035007 (2003) [arXiv:hep-ph/0211447]; G. Hiller et al., Phys. Lett. B 649, 152 (2007) [arXiv:hep-ph/0702191].

[19] G. Piranishvili, Ph.D. thesis, Dortmund Tech.U., August 2008.

[20] J. T. Wei et al. [Belle Collab.], Phys. Rev. D 78, 011101 (2008) [arXiv:0804.3656 [hep-ex]]. 